Negative results

\title{
H63D polymorphism in HFE is not associated with amyotrophic lateral sclerosis
}

\author{
Wouter van Rheenen ${ }^{a}$, Frank P. Diekstra ${ }^{a}$, Perry T.C. van Doormaal ${ }^{a}$, Meinie Seelen ${ }^{a}$, Kevin Kenna ${ }^{b}$, \\ Russell McLaughlin $^{\mathrm{b}}$, Aleksey Shatunov ${ }^{c}$, David Czell ${ }^{\mathrm{d}}$, Michael A. van Es ${ }^{\mathrm{a}}$, Paul W.J. van Vught ${ }^{\mathrm{a}, 1}$, \\ Philip van Damme e, Bradley N. Smith ${ }^{\text {, }}$, Stefan Waibel ${ }^{\text {f }}$, H. Jurgen Schelhaas ${ }^{g}$, Anneke J. van der Kooi ${ }^{\text {h }}$, \\ Marianne de Visser ${ }^{h}$, Markus Weber ${ }^{d}$, Wim Robberecht ${ }^{\mathrm{e}}$, Orla Hardiman ${ }^{\mathrm{b}}$, Pamela J. Shaw ${ }^{\mathrm{i}}$, \\ Christopher E. Shaw ${ }^{c}$, Karen E. Morrison ${ }^{j}$, Ammar Al-Chalabi ${ }^{c}$, Peter M. Andersen ${ }^{k}$, Albert C. Ludolph ${ }^{\mathrm{f}}$, \\ Jan H. Veldink ${ }^{\mathrm{a}, 2}$, Leonard H. van den Berg ${ }^{\mathrm{a}, *, 2}$ \\ ${ }^{a}$ Department of Neurology, Rudolph Magnus Institute of Neuroscience, University Medical Center Utrecht, Utrecht, the Netherlands \\ ${ }^{\mathrm{b}}$ Department of Neurology, Beaumont Hospital and Biomedical Sciences Institute, Trinity College, Dublin, Ireland \\ ${ }^{c}$ King's College London, Medical Research Council Centre for Neurodegeneration Research, Department of Clinical Neuroscience, Institute of Psychiatry, London, UK \\ ${ }^{\mathrm{d}}$ Neuromuscular Diseases Unit, Kantonsspital St. Gallen, St. Gallen, Switzerland \\ e Laboratory of Neurobiology, Vesalius Research Center, VIB, Department of Neurology, University Hospital Leuven, Experimental Neurology and Leuven Research Institute for \\ Neurodegenerative Diseases, University of Leuven, Leuven, Belgium \\ ${ }_{\mathrm{f}}$ Department of Neurology, University of Ulm, Ulm, Germany

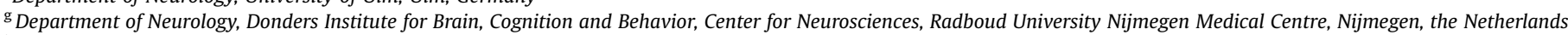 \\ h Department of Neurology, Academic Medical Center, University of Amsterdam, Amsterdam, the Netherlands \\ ${ }^{\mathrm{i}}$ Academic Neurology Unit, Department of Neuroscience, Faculty of Medicine, Dentistry and Health, University of Sheffield, Sheffield, UK \\ ${ }^{\mathrm{j} S}$ School of Clinical and Experimental Medicine, College of Medical and Dental Sciences, University of Birmingham, and University Hospitals Birmingham NHS Foundation Trust, \\ Birmingham, UK \\ ${ }^{\mathrm{k}}$ Department of Clinical Neuroscience, Umeå University, Umeå, Sweden
}

\section{A R T I C L E I N F O}

\section{Article history:}

Received 31 May 2012

Received in revised form 2 July 2012

Accepted 9 July 2012

\section{Keywords:}

Amyotrophic lateral sclerosis (ALS)

Motor neuron disease (MND)

HFE

H63D

rs 1799945

\begin{abstract}
A B S T R A C T
The H63D polymorphism in HFE has frequently been associated with susceptibility to amyotrophic lateral sclerosis (ALS). Regarding the role of HFE in iron homeostasis, iron accumulation is considered an important process in ALS. Furthermore, novel therapeutic strategies are being developed targeting this process. Evidence for this genetic association is, however, limited to several small studies. For this reason we studied the H63D polymorphism in a large European cohort including 3962 ALS patients and 5072 control subjects from 7 countries. After meta-analysis of previous studies and current findings we conclude that the H63D polymorphism in HFE is not associated with susceptibility to ALS, age at disease onset, or survival.
\end{abstract}

(c) 2012 Elsevier Inc. All rights reserved.

\section{Introduction}

Amyotrophic lateral sclerosis (ALS) is a devastating neurodegenerative disease affecting motor neurons in the brain and spinal cord. Patients suffer from progressive paralysis and generally succumb within 3 to 5 years after disease onset because of respiratory insufficiency (Hardiman et al., 2011). The etiology of ALS

\footnotetext{
* Corresponding author at: Department of Neurology, G03.228, University Medical Center Utrecht, P.O. Box 85500, 3508 GA Utrecht, the Netherlands. Tel.: +31 88 7557939; fax: +31302542100.

E-mail address: 1.h.vandenberg@umcutrecht.nl (L.H. van den Berg).

1 Present address: MRC-Holland, Amsterdam, the Netherlands.

2 Contributed equally to this work.
}

remains unknown and disease-modifying treatments are limited. A publicly available online meta-analysis of all genes studied in ALS (www.alsgene.org) identified the H63D polymorphism in HFE as one of the strongest susceptibility factors in ALS (Lill et al., 2010). Considering the role of HFE in iron homeostasis, iron accumulation is considered a possible mechanism contributing to ALS pathophysiology (Kwan et al., 2012; Rothstein, 2009). Moreover, this process has become a target for novel therapeutic strategies (Kupershmidt et al., 2011; Weinreb et al., 2011).

The studies that form the genetic basis for these strategies consistently report an increased risk of ALS for carriers of the H63D substitution (Goodall et al., 2005; He et al., 2011; Restagno et al., 2007; Sutedja et al., 2007; Wang et al., 2004). The individual studies 


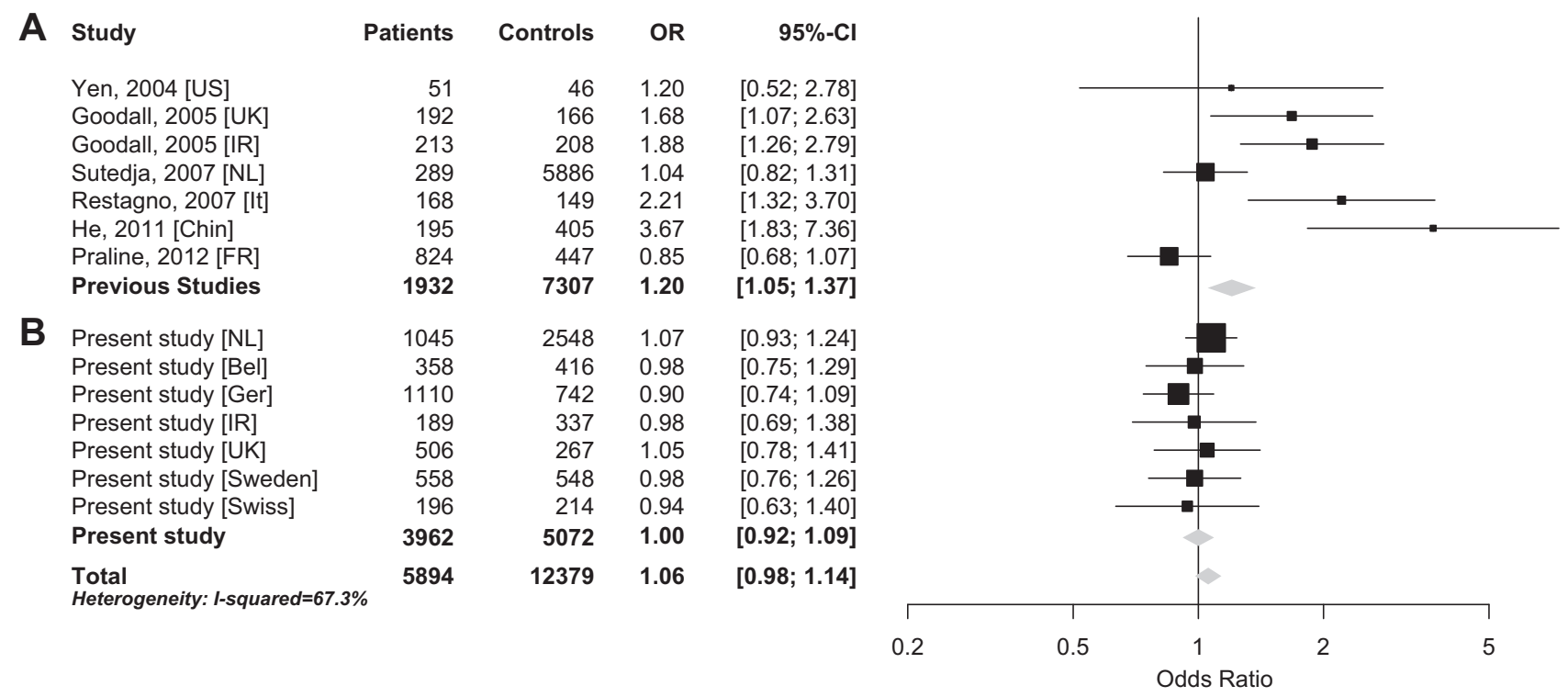

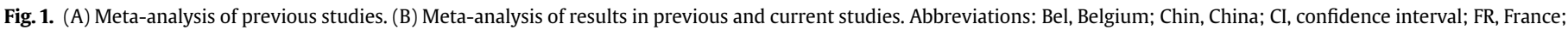
Ger, Germany; IR, Ireland; It, Italy; NL, the Netherlands; OR, odds ratio; UK, United Kingdom; US, United States.

are, however, moderately powered and as a result display wide confidence intervals for their effect size. Moreover, this association could not be replicated in a recent French study (Fig. 1A) (Praline et al., 2012).

For this reason we have conducted a genetic association study in a large European cohort including 7 different populations to definitely determine whether the H63D polymorphism in HFE is indeed associated with ALS.

\section{Methods}

Patients were recruited from outpatient clinics specializing in neuromuscular diseases at the participating centers from 7 different countries (the Netherlands, Belgium, Germany, United Kingdom, Ireland, Sweden, and Switzerland). Control subjects were unrelated individuals, free of any neuromuscular disease. We genotyped patients and control subjects using a predesigned TaqMan assay (Applied Biosystems, Foster City, CA, USA). The association between the H63D polymorphism and ALS was evaluated by logistic regression, correcting for country. An effect on age at onset was evaluated using linear regression, correcting for site of onset (spinal vs. bulbar) and sex. We tested the association with survival in a Cox proportional hazards model correcting for age at onset, site of onset, and sex.

\section{Results}

In total we included 3962 ALS patients and 5072 healthy control subjects (Supplementary Table 1 ). There was no difference in the H63D allele frequency between patients and control subjects (Supplementary Table 2; odds ratio, 1.00; 95\% confidence interval, 0.92-1.09). Combining our data with those of previous studies (5894 patients and 12,379 control subjects) again did not result in a significant association (Fig. 1B; odds ratio, 1.06; 95\% confidence interval, 0.98-1.14). We did not observe an effect of the H63D polymorphism on age at onset ( $p=0.62$ and $p=0.68$ for wild-type/ H63D and H63D/H63D genotypes respectively). Additionally, there was no effect on survival ( $p=0.26$ and $p=0.51$ for wild-type/H63D and H63D/H63D genotypes respectively; Supplementary Fig. 2).

\section{Discussion}

In a well-powered (99.98\% to detect the previously described effect) cohort we did not observe the association between the H63D polymorphism and ALS.

With the exception of a recent French study, these results contrast sharply with earlier studies on HFE in ALS, reporting a considerable effect size (Goodall et al., 2005; He et al., 2011; Praline et al., 2012; Restagno et al., 2007; Sutedja et al., 2007; Wang et al., 2004). Because our study population was derived from 7 different populations, including those from Ireland and the United Kingdom in which an association was previously found, a population-specific association cannot explain our findings (Goodall et al., 2005). Most likely, previous studies have suffered from publication bias favoring representation of a positive association. This bias is even more pronounced in smaller studies. A coincidental positive association in these studies has a higher chance of publication, and studies that fail to find an association will be criticized for lack of statistical power. This kind of bias highlights the importance of well-powered genetic association studies regardless of the association found.

In view of our findings, the perceived role of altered iron metabolism in ALS might need to be revised. Although there are data from imaging studies that suggest iron accumulation in ALS, and biochemical studies that indicate the presence of abnormal iron ligands in cerebrospinal fluid, the mechanisms of these changes remain to be elucidated, and it is possible that they are secondary rather than primary events in ALS (Ignjatovic et al., 2012; Kwan et al., 2012).

In conclusion, our data show that the H63D polymorphism in HFE is not a risk factor for ALS. The role of HFE in ALS pathogenesis, therefore, needs to be reconsidered. Furthermore, our data highlights the importance of large-scale genetic association studies in ALS.

\section{Disclosure statement}

The authors report no conflict of interest.

All participants gave written informed consent and the Medical Ethics Review Boards of the participating institutions approved this study. 


\section{Acknowledgements}

The research leading to these results has received funding from the European Community's Health Seventh Framework Programme (FP7/2007-2013) (grant agreement no. 259867), and the Motor Neurone Disease Association (MNDA). L.H.B., J.H., and A.J.K. receive support from Thierry Latran Foundation. L.H.B received travel grants and consultancy fees from Baxter International, Prinses Beatrix Fonds, and Biogen Idec, serves on the editorial board of Amyotrophic Lateral Sclerosis, and receives research support from the Netherlands Organisation for Health Research and Development, Prinses Beatrix Fonds, the Netherlands ALS Foundation, VSB Fonds, and Adessium Foundation.

\section{Appendix A. Supplementary data}

Supplementary data associated with this article can be found, in the online version, at http://dx.doi.org/10.1016/j.neurobiolaging. 2012.07.020. 